\title{
A Review of Fundamental Characteristics of Major Antenna
}

\author{
Rajesh Shrivastava*, Namita Pandey ${ }^{*}$, Ashutosh Pandey ${ }^{* *}$, K.N. Singh ${ }^{* *}$, \\ A.K. Shrivastava**, Komal Tamrakar** \\ * Research Scholar, Dr.C.V.Raman University Kota, Bilaspur, (C.G.), INDIA. \\ **Department of Physics , Dr.C.V.Raman University Kota , Bilaspur, (C.G.), INDIA.
}

\begin{abstract}
An Antenna is an essential terminal device in all types of communication and radar system. All communication and radar system requires an antenna in one form or the other. Without an antenna there would be no communication system and no radar system. The antenna is a source as well as a sensor of electromagnetic wave. In this paper an attempt has been made to receive the fundamental characteristics of different types of antenna with diagram. Further again it has been elaborated comprehensively its applications.

Keywords: device, communication, radar, electromagnetic wave, sensor.
\end{abstract}

\section{Introduction}

Antenna or aerial means the same. It is used in several domestic, civilian and military communication and radar application. It is also used in microwave radiation therapy system. An antenna is a transducer an impedance matching device, a radiator and a sensor of electromagnetic wave. It is an essential device/element in all types of communication and radar system. It can be considered as a source of electromagnetic wave. Again it has been seen that an antenna is a transducer between a guided wave and a radiated wave or vice-versa. The structure that "guides" the energy to the antenna is most evident as coaxial cable attached to the antenna. The radiated energy is characterized by the antennas' radiation pattern.

\section{Characteristics of Antenna}

An antenna is chosen for a particular application according to its main physical and electrical characteristics. Further an antenna must perform in a desired manner for the particular application. An antenna can be characterized by the following factors, not all are applicable to all types of antenna. Most of the characteristics mentioned below-

\subsubsection{Radiation resistance}

1.2.2 Radiation pattern

1.2.3 Beam Width

1.2.4 Band Width

1.2.5 Gain and main lobe

1.2.6 Front to back ratio

1.2.7 Aperture

1.2.8 The polarization of electric field

1.2.9 Lobes

1.2.10 Isotropic radiator

1.2.11 VSWR

1.2.12 VSWR bandwidth

1.2.13 Effective length

1.2.14 Radiation intensity

1.2.15 Antenna Efficiency

1.2.16 Antenna Impedance

1.2.17 Directive gain

1.2.18 Power gain

1.2.19 Effective area

1.2.20 Reciprocity

\subsubsection{Antenna Temperature}

1.2.1 Radiation resistance- $\left(\mathrm{R}_{\mathrm{r}}\right)$ : It is defined as a fictitious or hypothetical resistance that would be dissipate an amount of power equal to the radiation power.

$$
\mathrm{R}_{\mathrm{r}}=\text { Power radiated } / \mathrm{I}^{2}
$$


1.2.2 Radiation pattern: It is also called directional characteristics or radiation characteristics or antenna pattern. It is the graphical representation of radiation properties of the antenna as a function of space. That is the antennas' pattern describe how the antenna radiates energy out into space or how the antenna receive energy. It is important to state that an antenna radiates energy in all direction at least to some extent so, the antenna pattern is equally three dimensional. It is common, however, to describe this 3D pattern will two planner pattern, called the principle plane pattern. These are two types-

1- Field strength pattern

2- Power pattern

Field strength pattern- It is the variation of the absolute value of field strength as a function of $\theta$.

$\mathrm{EV}_{\mathrm{S}} \theta$ is called field strength pattern.

Power pattern- It is the variation of radiated power with $\theta$.

$\mathrm{PV}_{\mathrm{S}} \theta$ is called power pattern.

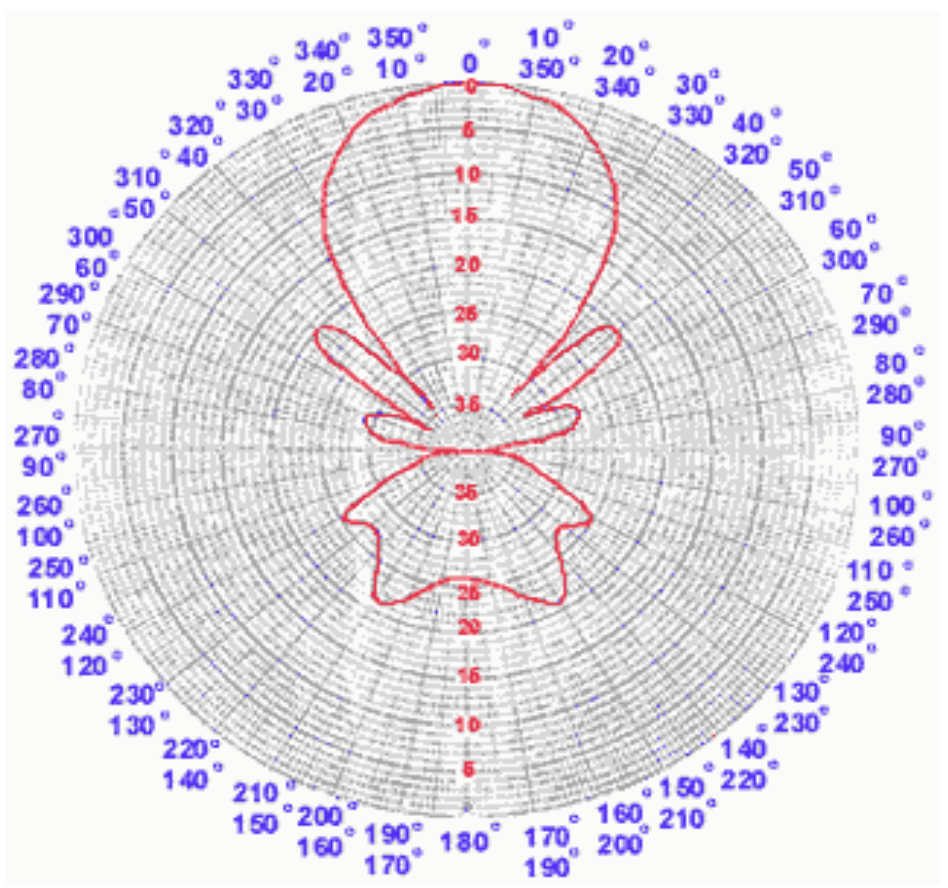

1.2.3 Beam width- The beam width (or half power beam width) of an antenna is typically defined for each of the principle plane as the angle between the point in the main lobes that are done from the maximum gain by $3 \mathrm{~dB}$.

S-Parameter [Magnitude in $\mathrm{dB}$ ]

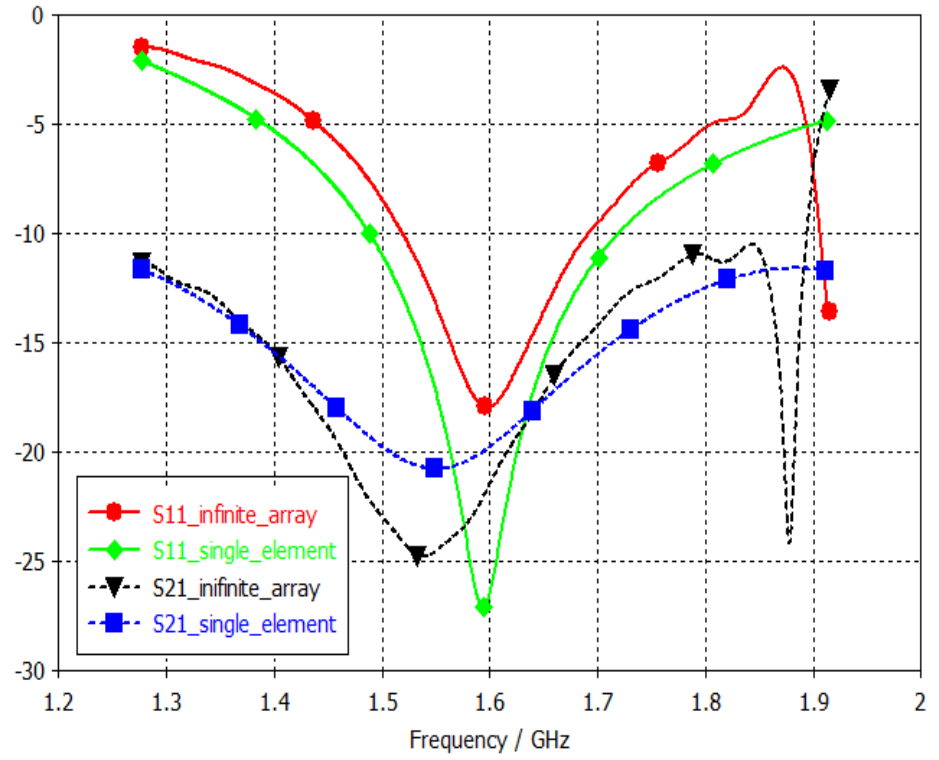


1.3.4 Band width- It is defined as the range of frequency over which the antenna maintained its characteristics and parameters, like gain, front and back ratio, standing wave ratio, radiation pattern polarization, impedance, directivity and so on without considerable change.

$|\mathrm{S} 1,1|$ versus Passes in $\mathrm{dB}$

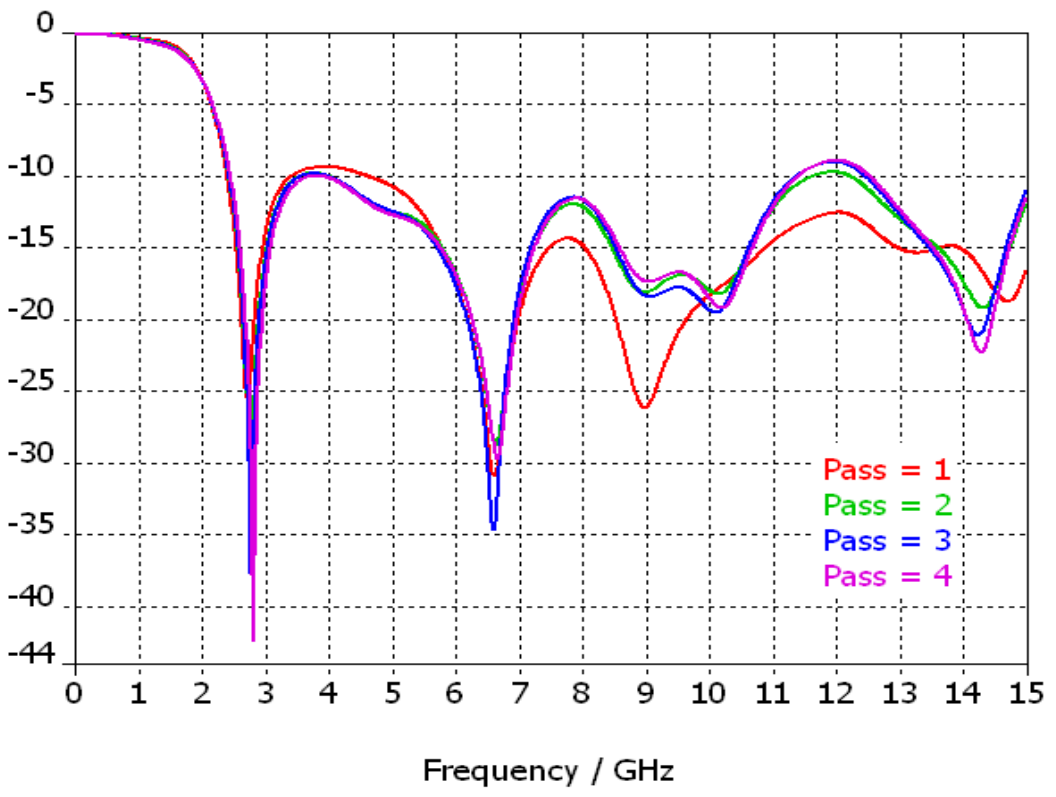

1.2.5 Gain of main lobe- The radiation pattern of an antenna shows the power on the bore sight as $0 \mathrm{~dB}$ and the power in other directions as negative value. The gain in other direction as negative values. The gain in all directions is plotted relative to the gain on bore sight.

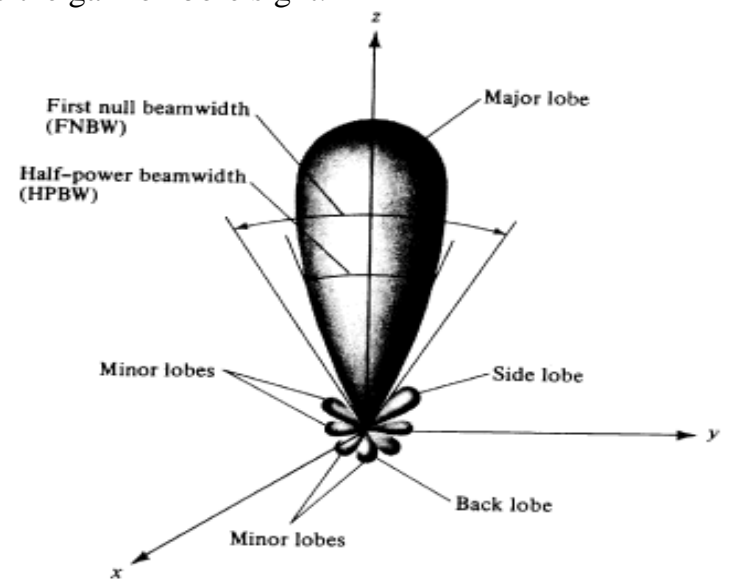

In order to find the absolute gain in any direction the gain on bore sight must be known. If this gain is expressed in decibels then this value can simply be added to the gain at any point to gain the absolute gain. The absolute gain on bore sight is measured by comparison with a standard gain antenna, which functions as a reference antenna whose gain is calculated or measured with a high degree of accuracy.

1.2.6 Front and back ratio- FBR is defined as the ratio of radiated power in the desired direction to the radiated power in the opposite direction.

That is,

$\mathrm{FBR}=$ radiated power in desired direction / radiated power in opposite direction.

1.2.7 Aperture- It is also called as capture area. In simple words aperture or capture area of antenna is the effective receiving area of the antenna and may be calculated from the power received and its comparison with the power density of the signal being received.

If, 
$\mathrm{S}=$ power density of the wave in watts/sq meter.

$\mathrm{A}=$ capture area of the antenna

$\mathrm{P}=$ total power absorbed by the antenna

Then,

$\mathrm{P}=\mathrm{S}$.A watts of $\mathrm{A}=\mathrm{P} / \mathrm{S}$.

The aperture of the antenna governs the size of its beam width. In general, the larger the aperture the narrower the beam width and the higher is the gain at a gain frequency.

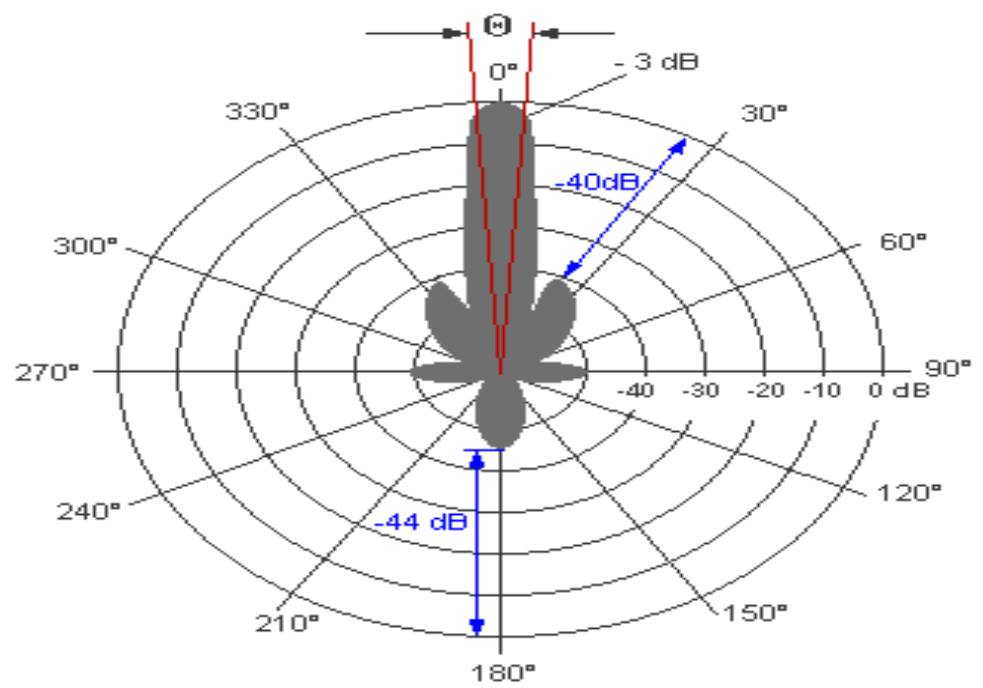

1.2.8 Polarization It is defined as the direction of the electric vector of the EM wave produced by an antenna. Polarization is used almost exclusively to describe the shape and orientation of the locus of the extremity of the electric field vector as its varies with time at a fixed point in space. This locus would be straight line an ellipse or a circular. It is of three types-

1. linear polarization

2. circular polarization

3. Elliptical polarization

1.2.9 Lobes- Any given antenna pattern has portions of the pattern that are called lobes. A lobe can be a main lobe, a side lobe, or a back lobe and these description refer to that portion of the pattern in which the lobe appears. In general, a lobe is any part of the pattern that is surrounded by regions of relatively weaker radiations. So a lobe is any part of the pattern that"sick out" and the names of the various types of lobes are somewhat self explanatory. Figure $\mathrm{X}$ and $\mathrm{Y}$

Provides a view of a radiation pattern with the lobes labeled in each type of the plot.

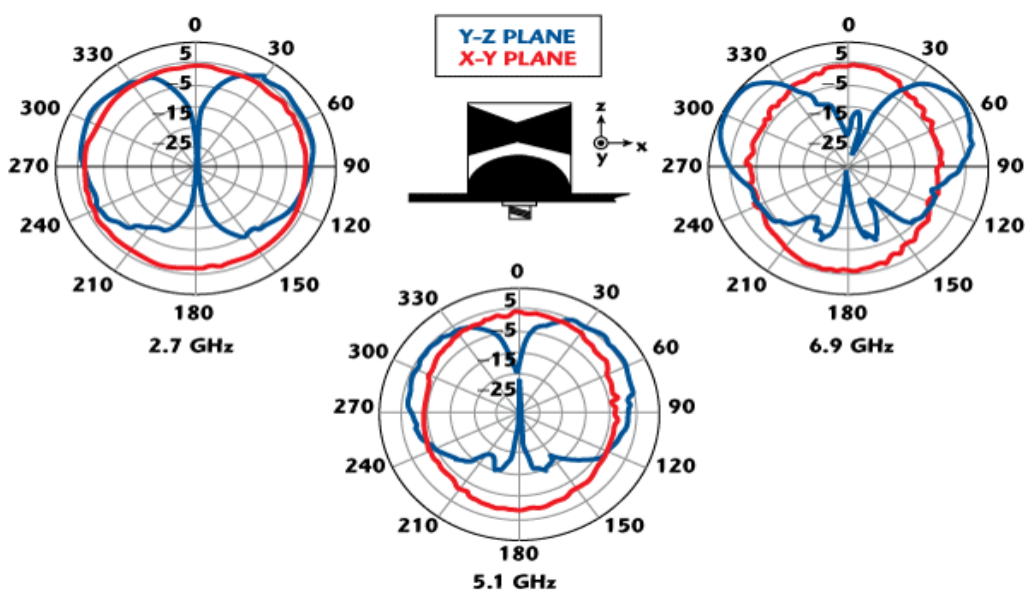


1.2.10 Isotropic radiation- An isotropic radiation is a hypothetical loss less antenna that radiates its energy equally in all directions. This imaginary antenna would have a spherical radiation pattern and the principle plane cuts would both be circles (indeed, any plane cut would be circle).

1.2.11 VSWR- The voltage standing wave ratio (VSWR) is defined as the ratio of the maximum voltage to the minimum voltage in a standing wave pattern. A standing wave is developed when power is reflected from a load. So the VSWR is a measure of how much power is delivered to a device as opposed to the amount of power that reflected from the device. If the source and the load impedance are the same, the VSWR is 1:1 there is reflected power. If VSWR is also measure of how closely the source and load impedance are matched. For most antennas' in WLAN it is measure of how close the antenna is to a perfect $50 \mathrm{ohms}$.

1.2.12 VSWR Bandwidth- The VSWR bandwidth is defined as the frequency range over which an antenna has a specified VSWR. Often the 2:1 VSWR bandwidth is specified, but 1:5:1 is also common.

1.2.13 Effective length- $\left(L_{\text {eff }}\right)$ It is used to indicate the effectiveness of the antenna as a radiators or receivers of EM energy.

$\mathrm{L}_{\text {eff }}$ of transmitting antenna- It is equal to the length of an equivalent linear antenna which radiates the same field strength as the actual antenna and the current is constant throughout the length of the linear antenna.

$\mathrm{L}_{\text {eff }}$ of transmitting antenna is defined mathematically as ,

$$
\mathrm{L}_{\text {eff }}\left(\mathrm{T}_{\mathrm{x}}\right)=1 / \mathrm{I}_{\mathrm{c}}
$$

$\mathrm{L}_{\text {eff }}$ of receiving antenna-

$$
\mathrm{L}_{\text {eff }}\left(\mathrm{R}_{\mathrm{x}}\right)=\operatorname{Voc} / \mathrm{E}(\mathrm{m})
$$

Effective length of an antenna is always less than the actual length. That is $\mathrm{L}_{\text {eff }}<\mathrm{L}$.

1.1.14 Radiation intensity- (RI) It is defined as the power radiates in a given direction per unit solid angle. That is ,

$\mathrm{RI}=\mathrm{r}^{2} \mathrm{pr}_{\mathrm{E}}^{2}{ }^{2} / \eta \quad$ watts/unit solid angle.

Here,

$$
\begin{aligned}
& \eta=\text { intrinsic impedance } \\
& r=\text { radius of the sphere } \\
& p=\text { power radiated instantaneously } \\
& E=\text { Electric field strength } \\
& \mathrm{RI}=\operatorname{RI}(\theta, \varphi) \text { is function of } \theta, \varphi
\end{aligned}
$$

1.2.15 Antenna Efficiency- $(\eta)$ It is defined as the ratio of the radiated power in the input power, That is

$$
\eta=\mathrm{w}_{\mathrm{r}} / \mathrm{w}_{\mathrm{t}}=\mathrm{w}_{\mathrm{r}} / \mathrm{w}_{\mathrm{t}}+\mathrm{w}_{\mathrm{l}}=\mathrm{g}_{\mathrm{p}} / \mathrm{g}_{\mathrm{d}}
$$

1.2.16 Antenna Impedance- Antenna Impedance is presented as the ratio of voltage to the current at the antennas terminals. Low and high frequency models are presented for transmission lines. The fundamentals of antenna theory requires that the antenna be "impedance matched" to the transmission line or the antenna will not radiate. 


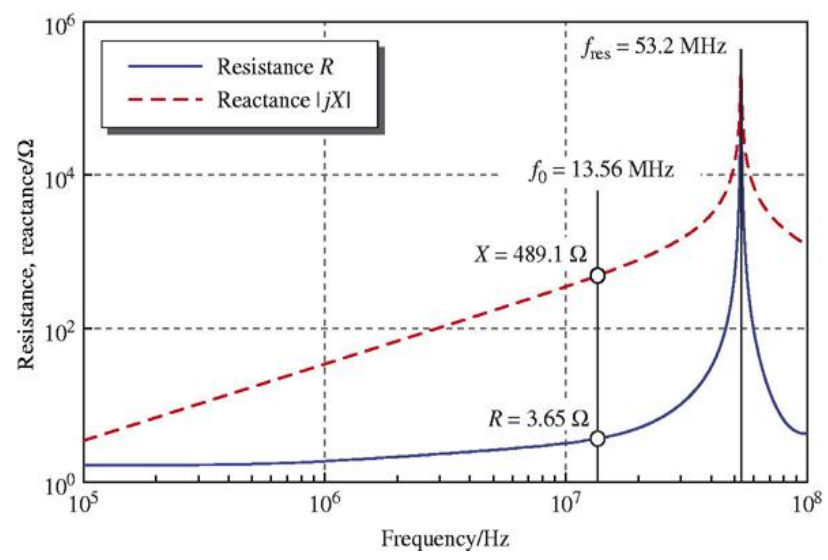

1.2.17 Directive gain- $\left(g_{d}\right)$ It is defined as the ratio of intensity of radiation in a specified direction to the average radiation intensity. That is,

$$
\begin{gathered}
\mathrm{g}_{\mathrm{d}}=\mathrm{RI} / \mathrm{RI}_{\mathrm{av}}=\mathrm{RI} .4 \pi / \mathrm{w}_{\mathrm{r}} \\
\mathrm{w}_{\mathrm{r}}=\text { radiated power }
\end{gathered}
$$

1.2.18 Power gain- $\left(g_{p}\right)$ It is defined as the ratio of $4 \pi$ times radiation intensity to the total input power. That is,

where $w_{t}=w_{r}+w_{1}$

$$
\mathrm{g}_{\mathrm{p}}=4 \pi .(\mathrm{RI}) / \mathrm{w}_{\mathrm{t}}
$$

$\mathrm{w}_{\mathrm{l}}=$ ohmic loss in the antenna

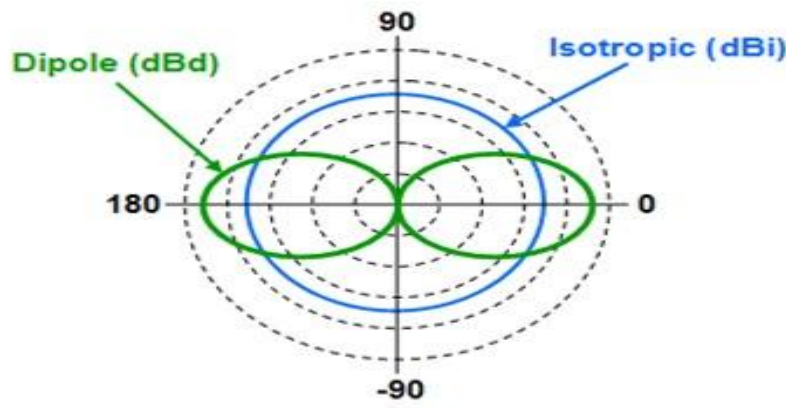

1.2.19 Effective Area- It is defined as

Where $\mathrm{w}_{\mathrm{R}}=$ received power $($ watt $)$

$$
\begin{gathered}
A_{e}=\left(\lambda^{2} / 4 \pi\right), g_{d}\left(m^{2}\right) \\
\text { Or } A_{e}=\left(w_{R} / P\right) m^{2}
\end{gathered}
$$

$\mathrm{P}=$ power flow per square meter for incident wave.

1.2.20 Reciprocity- It is a fundamental property of antennas that the electrical characteristics of an antenna described in the next section, such as gain, radiation pattern, impedance, band width, resonant frequency and polarization, are the same whether the antenna is transmitting or receiving.

1.2.21 Antenna Temperature- Antenna temperature is a property of an antenna and the environment it operate $\mathrm{in}$. It is a measure of the noise received by antenna due to thermal (or temperature) effects.

\section{Classification}

Antenna can be classified on the basis of-

1. Frequency

2. Aperture

3. Polarization

4. Radiation 
Classification of antenna based on frequency is as follows-

1. VLF antenna

2. LF antenna

3. HF antenna

4. VHF antenna

5. Microwave antenna

Microwave antenna-An antenna which is operated at microwave frequency is known as microwave antenna.

There are various types of microwave antenna and they are used in various application.

Classification of microwave antenna-

1. Microstrip patch antenna

2. Horn antenna

3. Parabolic antenna

4. Plasma antenna

5. MIMI antenna

In other words we can say that there are various types of antenna such as:-

1. Short dipole antenna

2. Dipole antenna

3. Half wave dipole antenna

4. Broadband dipole antenna

5. Monopole antenna

6. Folded dipole antenna

7. Loop antenna

8. Helical antenna

9. Yagi-Uda antenna

10. Spiral antenna

11. Corner reflector antenna

12. Parabolic reflector antenna( Dish antenna)

13. Rectangular Microstrip antenna( Patch antenna)

14. Planner inverted-F antenna

15. Bow Tie antenna

16. Log- Periodic Antenna

17. Log-Periodic Dipole Array

18. Slot antenna

19. Cavity backed antenna

20. Inverted-F antenna

21. Slotted waveguide antenna

22. Horn antenna

23. Vivaldi antenna

24. Telescopic antenna

25. NFC antenna

26. Fractal antenna

27. Wearable antenna

28. Microstrip Antenna

29. Plasma Antenna

$30 \quad$ CNT Antenna

$31 \quad$ Long Wire Antenna

32 V Antenna

33 Rhombic Antenna

34 Beverage Antenna

35 Slotted Cylindrical Antenna

36 Septum Antenna

\section{Short dipole antenna-}

The short dipole antenna is simplest in all types of antenna. It is simply open circuited wire fed at its centre as given in following figure. 


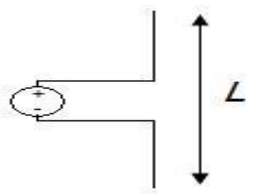

02 Dipole antenna-

Dipole antenna with very thin radius is considered. It is similar to short dipole except it is not required to be small compared to the wavelength.

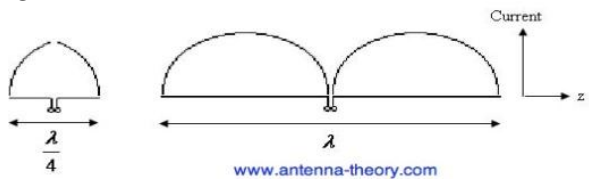

\section{Half wave dipole antenna-}

The half-wave dipole antenna is just a special case of the dipole antenna, but it's important enough that it will have its own section. Note that the "half-wave" term means that the length of this dipole antenna is equal to a half-wavelength at the frequency of operation.

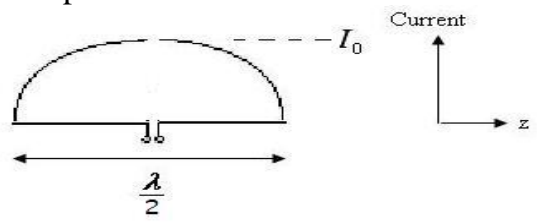

04 Broadband dipole antenna-

A standard rule of thumb in antenna design is: an antenna can be made more broadband by increasing the volume it occupies. Hence, a dipole antenna can be made more broadband by increasing the radius $A$ of the dipole.

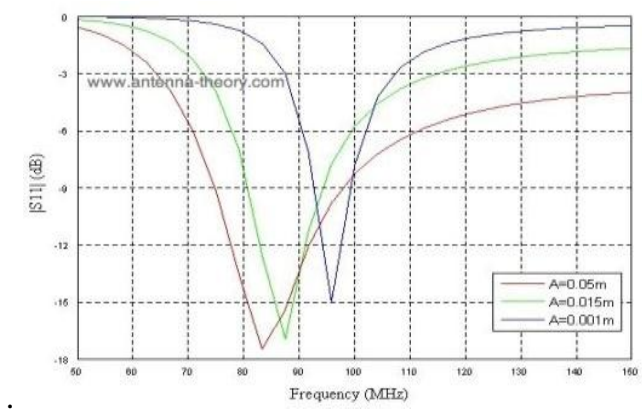

\section{Monopole antenna-}

A monopole antenna is one half of a dipole antenna, almost always mounted above some sort of ground plane. The case of a monopole antenna of length $L$ mounted above an infinite ground plane is shown in Figure-

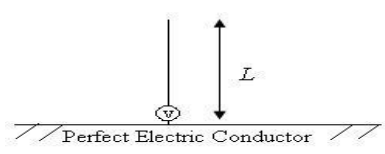

(a)

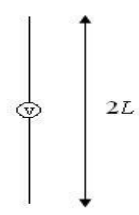

(b)

06 Folded dipole antenna-

A folded dipole is a dipole antenna with the ends folded back around and connected to each other, forming a loop as shown in Figure- 


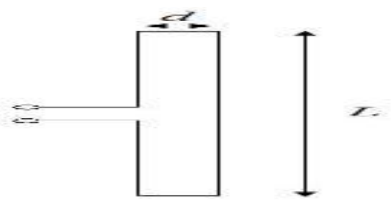

\section{Loop antenna-}

The small loop antenna is a closed loop as shown in Figure 1. These antennas have low radiation resistance and high reactance, so that their impedance is difficult to match to a transmitter. As a result, these antennas are most often used as receive antennas, where impedance mismatch loss can be tolerated.-

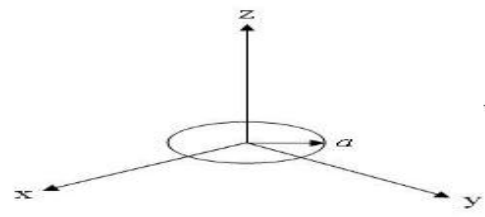

08 Helical antenna-

The most popular helical antenna (helix) is a travelling wave antenna in the shape of a corkscrew that produces radiation along the axis of the helix antenna. These helix antennas are referred to as axial-mode helical antennas. The benefits of this helix antenna is it has a wide bandwidth, is easily constructed, has a real input impedance, and can produce circularly polarized fields. The basic geometry of the helix antenna shown in Figure

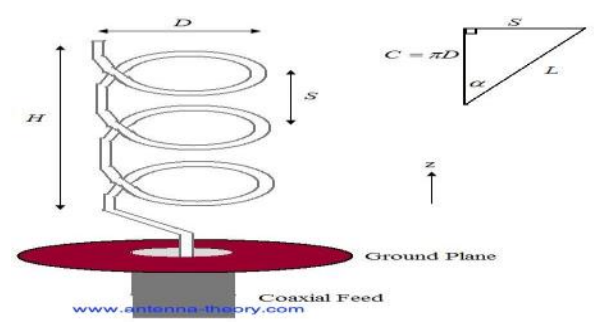

\section{Yagi-Uda antenna-}

The Yagi-Uda antenna or Yagi Antenna is one of the most brilliant antenna designs. It is simple to construct and has a high gain, typically greater than $10 \mathrm{~dB}$. The Yagi-Uda antennas typically operate in the HF to UHF bands (about $3 \mathrm{MHz}$ to $3 \mathrm{GHz}$ ), although their bandwidth is typically small, on the order of a few percent of the center frequency. You are probably familiar with this antenna, as they sit on top of roofs everywhere. An example of a Yagi-Uda antenna is shown below.

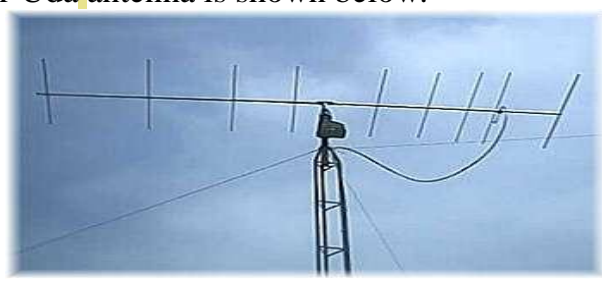

\section{Spiral antenna-}

Spiral antennas belong to the class of "frequency independent" antennas; those with a very large bandwidth. The fractional Bandwidth can be as high as 30:1. This means that if the lower frequency is 1 $\mathrm{GHz}$, the antenna could still be in band at $30 \mathrm{GHz}$, and every frequency in between.-

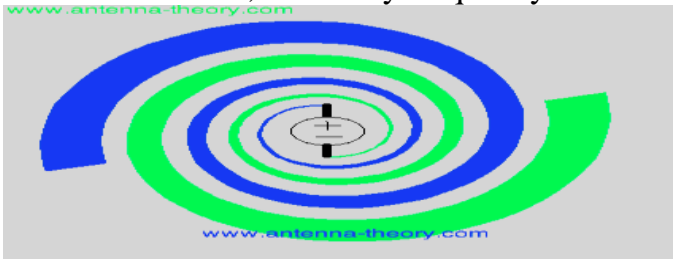

\section{Corner reflector antenna-}

To increase the directivity of an antenna, a fairly intuitive solution is to use a reflector. For example, if we start with a wire antenna (lets say a half-wave dipole antenna), we could place a conductive sheet behind it to 
direct radiation in the forward direction. To further increase the directivity, a corner reflector may be used, as shown in Figure 1. The angle between the plates will be 90 degrees.-

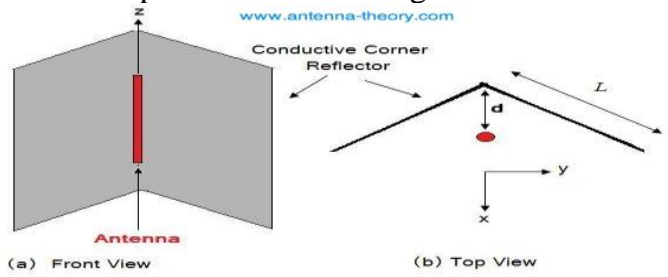

12 Parabolic reflector antenna( Dish antenna)-

The most well-known reflector antenna is the parabolic reflector antenna, commonly known as a satellite dish antenna. Examples of this dish antenna are shown in the following figures.

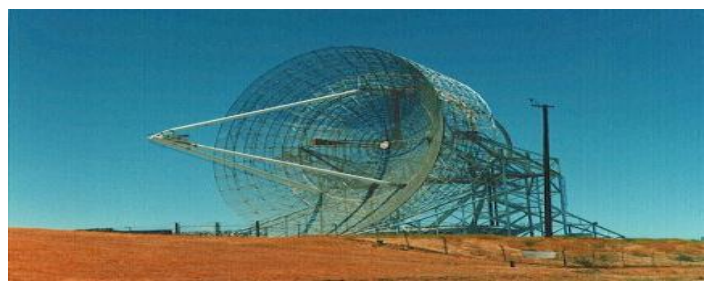

\section{Rectangular Microstrip antenna( Patch antenna)-}

Microstrip or patch antennas are becoming increasingly useful because they can be printed directly into a circuit board. Microstrip antennas are becoming very widespread within the mobile phone market. Patch antennas are low cost, have a low profile and are easily fabricated.

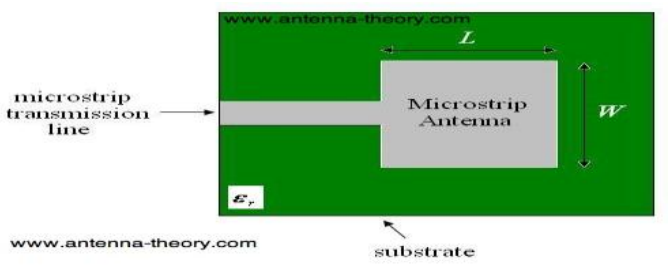

\section{Planner inverted-F antenna-}

Antenna designers are always looking for creative ways to improve performance. One method used in patch antenna design is to introduce shorting pins (from the patch to the ground plane) at various locations. To illustrate how this may help, two instances will be illustrated, the quarter-wavelength Patch Antenna, which leads into the Planar Inverted-F Antenna (PIFA).

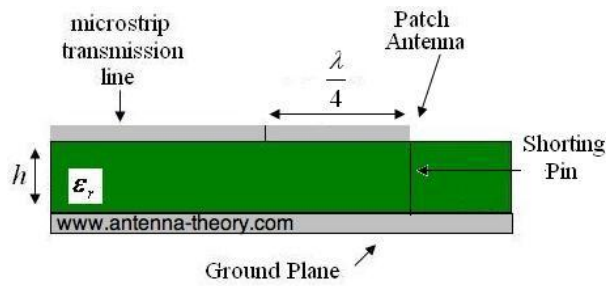

\section{Bow Tie antenna-}

Bow-Tie antennas aren't really Log Periodic antennas; however, the bow tie (or butterfly) antenna makes a good starting point to begin talking about Log-Periodic antennas. So we will first discuss properties of wideband antennas, and then discuss the infinite bow-tie antenna, and then measurements and properties of the real bow-tie antenna.- 


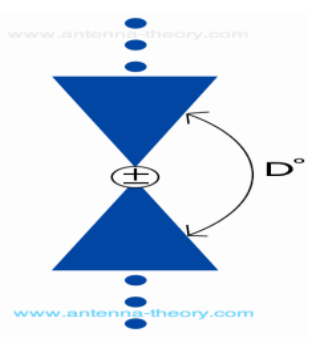

\section{Log- Periodic Antenna-}

Log-Periodic antennas antennas are designed for the specific purpose of having a very wide bandwidth. The achievable bandwidth is theoretically infinite; the actual bandwidth achieved is dependent on how large the structure is (to determine the lower frequency limit) and how precise the finer (smaller) features are on the antenna (which determines the upper frequency limit). On this page, we will expand on the ideas related to wideband antennas presented on the Bow Tie Antennas) page. Then we will explain why Log-Periodicity in antennas will produce wide bandwidth.-

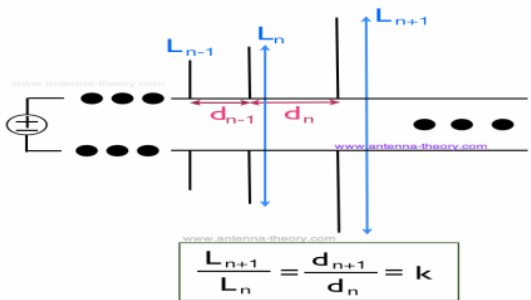

\section{Log-Periodic Dipole Array-}

This antenna is often characterized by "active" and "passive" regions. This means that if we are discussing the radiation mechanism at say $f=300 \mathrm{MHz}$, then the bulk of the radiation from this antenna will come from the dipoles with lengths near half a wavelength at $300 \mathrm{MHz}$ (so $L=0.5$ meters). This is illustrated in Figure.

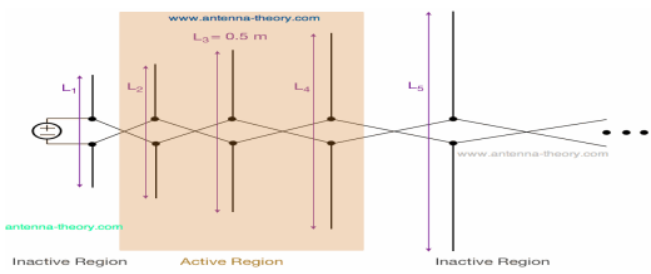

\section{Slot antenna-}

Slot antennas are used typically at frequencies between $300 \mathrm{MHz}$ and $24 \mathrm{GHz}$. The slot antenna is popular because they can be cut out of whatever surface they are to be mounted on, and haveradiation patterns that are roughly omnidirectional (similar to a linear wire antenna, as we'll see). The polarization of the slot antenna is linear. The slot size, shape and what is behind it (the cavity) offer design variables that can be used to tune performance.

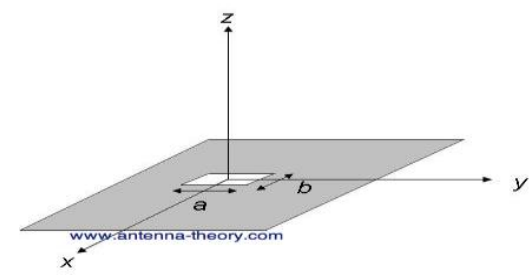

\section{Cavity backed antenna-}

The basic cavity-backed slot antenna is shown in Figure 1 (in a rectangular cube of size $\mathrm{A}^{*} \mathrm{~B} * \mathrm{C}$ ). The walls are metallic (electrically conducting), and the inside is hollow. On one end, a slot is cut out. The cavity is typically excited by a probe antenna in the intererior of the cavity, which typically is modelled as a monopole antenna. The exciting monopole antenna is shown in green.- 

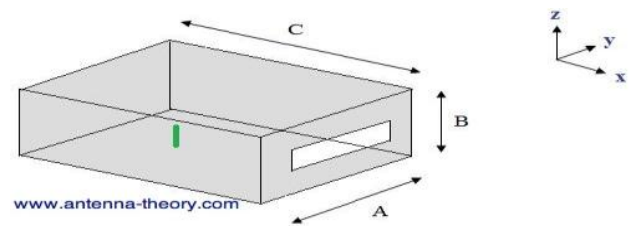

20 Inverted-F antenna-

The inverted-F antenna is shown in Figure. While this antenna appears to be a wire antenna, after some analysis of how this antenna radiates, it is more accurately classified as an aperture antenna.-

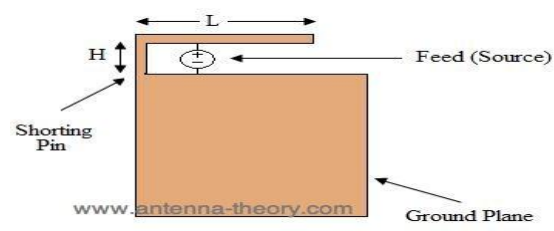

\section{Slotted waveguide antenna-}

Slotted antenna arrays used with waveguides are a popular antenna in navigation, radar and other highfrequency systems. They are simple to fabricate, have low-loss (high efficiency) and radiate linear polarization with low cross-polarization. These antennas are often used in aircraft applications because they can be made to conform to the surface on which they are mounted. The slots are typically thin (less than 0.1 of a wavelength) and 0.5 wavelengths long (at the center frequency of operation).

\section{Horn antenna-}

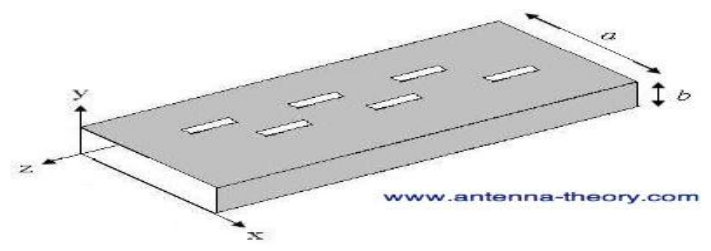

Horn antennas are very popular at UHF (300 MHz-3 GHz) and higher frequencies (I've heard of horn antennas operating as high as $140 \mathrm{GHz}$ ). Horn antennas often have a directional radiation pattern with a high antenna gain, which can range up to $25 \mathrm{~dB}$ in some cases, with 10-20 dB being typical. Horn antennas have a wide impedance bandwidth, implying that the input impedance is slowly varying over a wide frequency range (which also implies low values for $\underline{\mathrm{S} 11}$ or VSWR). The bandwidth for practical horn antennas can be on the order of 20:1 (for instance, operating from $1 \mathrm{GHz}-20 \mathrm{GHz}$ ), with a 10:1 bandwidth not being uncommon.

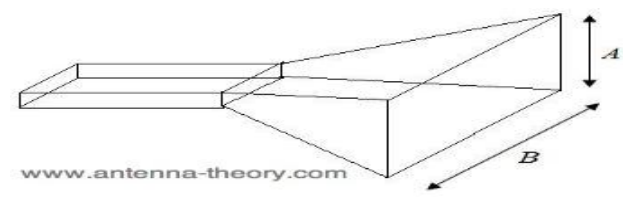

\section{Vivaldi antenna-}

Vivaldi antennas are simple planar antennas that are very broadband. The polarization is linear, and the basic antenna structure is shown in figure

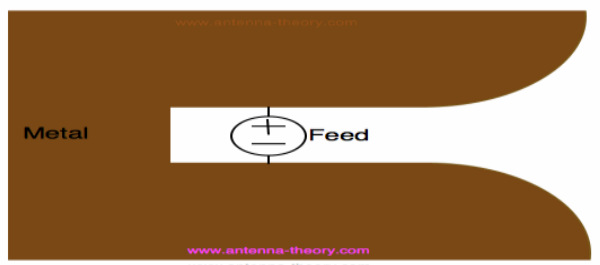

\section{Telescopic antenna-}

Telescopes aren't really antennas. Antennas are connected to a receiver or transmitter, and collect or transmit radiated electromagnetic waves. However, telescopes do collect electromagnetic waves - they just don't 
process them. Now, your eye does act as a receiver that processes the electromagnetic waves. When the telescope is placed up to the eye, it greatly increases the effective aperture of your eye, which means the peak antenna gain of your eye is significantly increased.

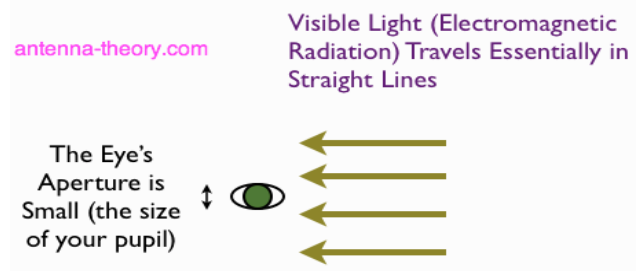

\section{NFC antenna-}

NFC stands for Near Field Communications and goes by the acronym NFC. NFC is simply a set of standards for smart phones or whatever to establish communication with each other by bringing them into close together (typically $0-5$ centimeters). This set of standards is just like $802.11 \mathrm{~b}$ or $802.11 \mathrm{n}$ for WIFI - it sets the protocols to send and receive information. The application of NFC include swiped proximity payments (such as google wallet for paying at Starbucks), information exchange at small distances (for instance, touching smartphones to share contact information), and simplified setup of devices such as Wi-Fi or Bluetooth. Communication is also possible between an NFC device and an unpowered NFC chip, called a tag (as in RFID tag).-

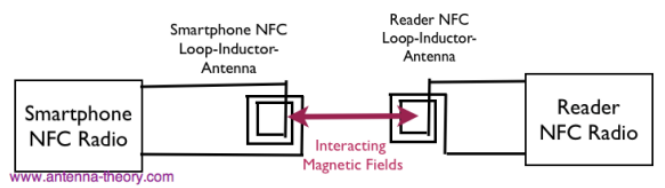

\section{Fractal antenna-}

Fractals are those fun shapes that if you zoom in or zoom out, the structure is always the same. They have wild properties, like having a finite area but infinite perimeter. They are often constructed via some sort of iterative mathematical rule, that generates a fractal from a simple object step by step. In the last section of this page, we will do that in designing a fractal antenna.-

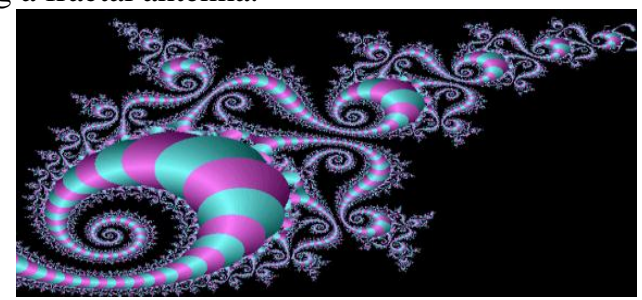

\section{Wearable antenna-}

Wearable Antennas are essentially any antenna that is specifically designed to function while being worn. Examples include smartwatches (which typically have integrated bluetooth antennas), glasses (such as Google Glass which has WIFI and GPS antennas), GoPro action cameras (which have wifi and bluetooth antennas, and are often strapped to a user to obtain their footage), and even the Nike+ Sensor (which communicates to a smartphone via bluetooth, and is placed in a user's shoe). Wearable antennas are becoming increasingly common in consumer electronics, and as such this page is dedicated to describing the unique difficulties involved in wearable antenna design.-

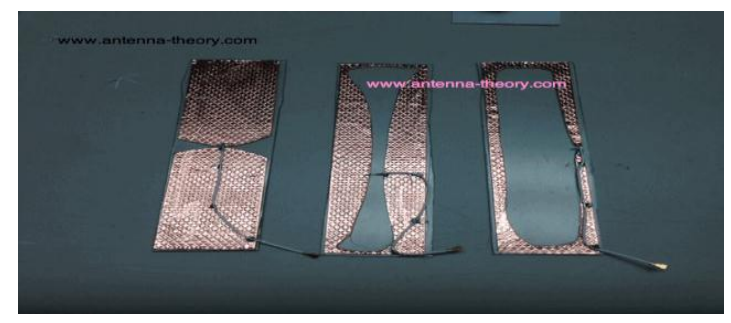

28 Microstrip Antenna-_Microstrip antenna are often used where thickness and conformability to the host surface are the key requirement. Since patch antenna can be directly printed onto acircuit board, these are 
becoming increasingly popular within the mobile phone market. They are low cast, have a low profile and are easily fabricated.

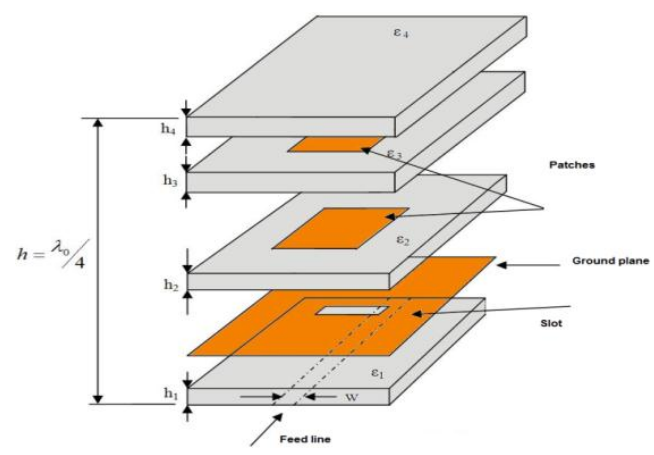

\begin{tabular}{ll}
\multicolumn{2}{c}{ Application of micro strip antenna- } \\
\hline 1. & Automatic toll collection \\
2. & Cellular phone \\
3. & Cellular video \\
4. & Collision avoidance radar \\
5. & Direct broad cast satellite \\
6. & Global positioning satellite \\
7. & GSM \\
8. & Paging \\
9. & Personal communication system \\
10. & Wide area computer network \\
11. & Wireless local area network
\end{tabular}

29 Plasma antenna-A plasma antenna is a column of ionized gas in which the free electron emit, Absorb and reflect radio signals just as the free electrons in a metal antenna. The plasma antenna can be made to appear and disappear in milliseconds. The plasma antenna has an adjustable high frequency cut off.

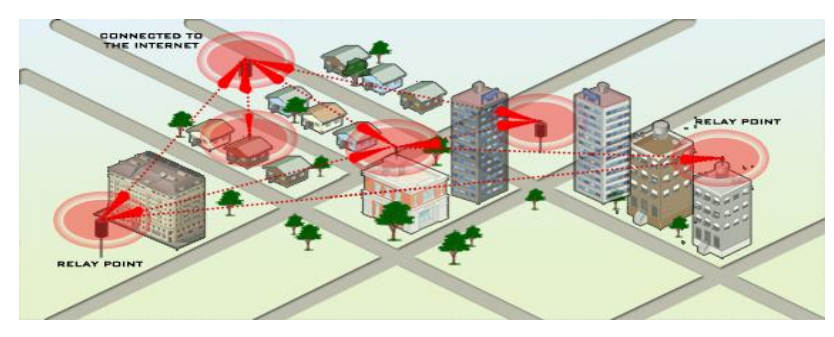

Applications-
1. High speed digital communication
2. Radar system
3. In radio antenna
4. $4 \mathrm{G}$
5. RFID
6. Digital Home

30 CNT antenna-Fundamental properties of dipole transmitting antenna formed by carbon nano tubes are investigated, since carbon nano tubes can be grown to centimeter length and since they can be metallic, the properties of carbon nano tubes as antenna elements are of fundamental interest. It was discovered in 1991. At an atomic level, grapheme has an honey comb structure. The small circles denote the location of carbon atoms and the lines depict carbon-carbon bond. 


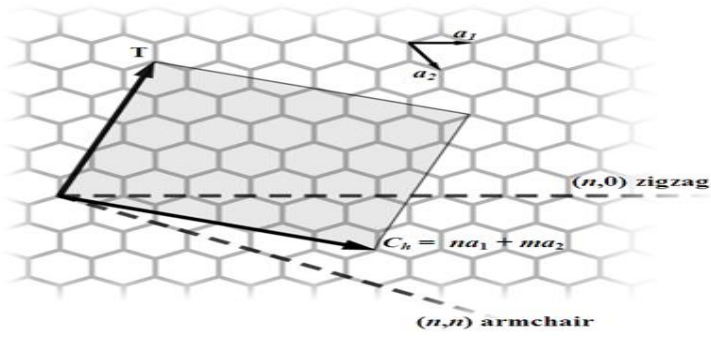

Electrically, carbon nano tubes have fascinating properties. For example, they can be either metallic or semiconducting depending on their geometry. Arm chair CN's are always metallic ( they exhibit no energy band gap) as are zigzag CN's with $\mathrm{m}=3 \mathrm{q}$, where $\mathrm{q}$ is integer. The special nature of the carbon nano tubes conductivity results in unique property for carbon nano tube antenna. First, it should be noted that nano electronic devices are inherently high impedance. For example at the nano scale dc electron transport is typical either ballistic exhibiting a quantized resistance on the order of $R_{0}=12.9 \mathrm{k} \Omega$ or via tunneling across gaps with as associated high tunneling resistance. Few more antennas are-

\section{Conclusion}

From the above elaborate study it has been concluded that an antenna is an essential part of communication as well as propagation. This paper gives details information about types of antenna \& application also contains the basics of antenna. It is found that, due to properties of the carbon nano tubes conductivity function, and its relationship to Plasmon effects, some properties of carbon nano tube antenna are quite different from the case of an infinitely thin copper antenna of the same size and shape. Important conclusion of this paper are that carbon nano tube antenna are found to exhibit Plasmon resonance above a sufficient frequency, have high input impedances ( which is probably beneficial for connecting to nano electronic circuits), and exhibit very low efficiencies.

\section{Acknowledgement}

The author is grateful to Head Dept. of Physics, Dr. C.V. Raman University, Kota Bilaspur (C.G.) India for providing facilities at the research centre, and reviewers for helpful comments for pointed out several pertinent references.

\section{References}

[1]. Pramod Dhandev," Antenna and its Application,” Armament Research and Development Establishment,Dr. Homi Bhabha Rd, Pashan.

[2]. J.R James \& P.S. Hall,” Handbook of Microstrip Antenna , Artech House Publication.

[3]. I. Lijima,' Helical Microtubels of Graphitic Carbon," Nature,vol. 354 pp.56-58,1991

[4]. S.Li,Z,Yu,C. Rutherglan, and P.J.Bruke," Electrical Properties of $0.4 \mathrm{~cm}$ Long Single Walled Carbon nano Tubes,” Nano let., vol. 4, pp. 2003-2007,2004

[5]. Z.Yao, C. Dekker and P Avouris, "Electrical TransportTthrough Single Walled Carbon Nano tubes," in Carbon nano tubes; oics in Applied Physics, M.S. Dresselhouse, G.Dresselhouse, and P. Avorious,Eds. Berlin, Germany,:Springer-Verlag,2001, vol. 80, pp. $147-171$

[6]. S. Dutta Electronic Transport in Masoscopic Systems. Cabridge, U.K.: Cambridge University Press,1995

[7]. S.Li.z.Yu.S.F. Yen, W.C. Tang and P.J. Bruke," Carbon nanotube Transistor operation at 2.6 GHz," Nano let. Vol. 4, pp. 753 756,2004

[8]. K.G. Ong, K. Zeng, C. A. Grimes,” A wireless,passive, Carbon Nanotube-VBased Gas Sensor,’IEEE Sensor J.,vol.2,pp.82-88,2002

[9]. P.Kim. and C.M. Lieber," Nanotubes Nanotweezers,” Science,vol.286,pp2184-2150,1999

[10]. G. Prio, P. Legagneux, D. Pribat, K.B.K. Teo, M. Chhowalla, G.A.J. Amaratunga and W.I. Milne," Fabrication and Electrical Charactrisctcs of Carbon Nanotubes field Emission Microcathodes with an Integrated Gate Electrode," Nanotechnology, vol. 13,pp. $1-4$.

[11]. G.S.N. Raju," Antenna and Wave Propagation”, Person Education India, 2005, pp. 137-142

[12]. John D Kraus,Ronald J Marhefka, Ahmed S Khan,” Antenna and Wave Propagation”, Tata McGraw Hill Education Pvt. Ltd.,2010, pp.517 